\title{
Analysis of cellular senescence in gingival tissues and gingival fibroblast cultures
}

\section{Masae Furukawa ( $\nabla$ masae@ncgg.go.jp )}

Department of Oral Disease Research, National Center for Geriatrics and Gerontology, Obu

\section{Kazunari Matsuda}

Biological \& Analytical Research Group, R\&D Department, Daiichi Sankyo Healthcare Co., Ltd.

\section{Mie Kurosawa}

Department of Oral Disease Research, National Center for Geriatrics and Gerontology, Obu

Jingshu Wang

Department of Oral Disease Research, National Center for Geriatrics and Gerontology, Obu

\section{Maki Watanabe}

Department of Oral Disease Research, National Center for Geriatrics and Gerontology, Obu

\section{Yu Aoki}

Biological \& Analytical Research Group, R\&D Department, Daiichi Sankyo Healthcare Co., Ltd.

\section{Yosuke Shikama}

Department of Oral Disease Research, National Center for Geriatrics and Gerontology, Obu

\section{Kenji Matsushita}

Department of Oral Disease Research, National Center for Geriatrics and Gerontology, Obu

\section{Research Article}

Keywords: aging, periodontal disease, hydrogen peroxide, chronic inflammation, proinflammatory cytokines

Posted Date: December 10th, 2020

DOl: https://doi.org/10.21203/rs.3.rs-121526/v1

License: (c) (1) This work is licensed under a Creative Commons Attribution 4.0 International License. Read Full License 


\section{Abstract}

Senescence in periodontal tissues may be involved in the pathogenesis of periodontitis. In this study, we analyzed cellular senescence in gingival tissues of aged mice and in gingival fibroblast cultures and investigated the relationship of cellular senescence with the pathogenesis of periodontal disease. The proportions of senescence-associated $\beta$-galactosidase (SA- $\beta$-gal)-positive cells and the mRNA expression levels of p16 and p21 in gingival tissues and gingival fibroblasts from 20-month-old mice were significantly higher than those in gingival tissues and gingival fibroblasts from 10 -week-old mice. The mRNA expression levels of IL-1 $\beta$ and TNF- $\alpha$ as well as the numbers of total macrophages and M1 macrophages were increased in gingival tissues of aged mice. Expression of these senescenceassociated molecules was also increased in long-term passaged or hydrogen peroxide-stimulated human gingival fibroblasts; however, it was significantly suppressed by treatment with the senolytic drug ABT263. These results suggest that cellular senescence can be induced in gingival fibroblasts in culture as was observed in gingival tissues of aged mice. An increase in senescent cells in the gingiva may be associated with the pathogenesis of periodontitis in part through activating an inflammatory response in periodontal tissues.

\section{Introduction}

Aging is the most powerful risk factor that is common to chronic diseases and increased mortality. ${ }^{1}$ It has been suggested that aging also contributes to the development of lifestyle-related diseases such as cardiovascular diseases and diabetes, and aging of cells in blood vessels and adipose tissue may be the basis of the pathogenesis of lifestyle-related diseases. ${ }^{2-5}$ Periodontitis is also a disease for which the incidence increases with aging, and aging is thought to be one of the risk factors of periodontitis. ${ }^{2}$ However, the relationship between aging of periodontal tissues and the pathogenesis of periodontitis has remained unclear. ${ }^{3}$

Senescence at the cellular level (cellular senescence) ${ }^{4}$ in organ tissues not only affects the senescence of individuals but may also be involved in the development of various age-related diseases. Cellular senescence is a phenomenon ${ }^{5}$ in which irreversible cell cycle arrest occurs, and it is caused by telomere shortening, regulation of oncogenes and tumor suppressor genes, and stresses due to radiation, ultraviolet rays, and reactive oxygen species. Senescent cells share common characteristics including cell cycle constancy, cell enlargement and flattening, formation of senescence-associated heterochromatic foci (SAHF), formation of DNA damage foci (DNA-SCARS), activation of senescence-associated $\beta$ galactosidase (SA- $\beta$-gal), and increased expression of cell cycle inhibitors p16 and p21. ${ }^{6}$ Senescent cells exhibit a phenomenon in which various inflammatory cytokines and various physiologically active substances such as matrix metalloproteases and growth factors (SASP: senescence-associated secretory phenotype) are secreted, and they function to maintain homeostasis such as homeostasis in wound healing. ${ }^{7-9}$ On the other hand, this phenomenon causes chronic inflammation in surrounding tissues, and it has been suggested that it is involved in the onset and progression of age-related diseases such as 
cancer, arteriosclerosis, chronic obstructive pulmonary disease, and Alzheimer's disease. ${ }^{10}$ Therefore, it is possible that an increase in senescent cells may be involved in the pathogenesis of periodontal disease, which is a chronic inflammatory disease. However, the relationship between senescence and inflammation in periodontal tissue remains unclear.

In this study, we clarified the dynamics of senescent cells in gingival tissues using an aged mouse model. Next, we investigated the induction of senescence in gingival fibroblasts and the associated expression of inflammation-related genes with the aim of determining the relationship between periodontitis and cellular senescence. ${ }^{10}$

\section{Materials And Methods}

\section{Cell culture}

Normal human gingival fibroblasts (HGFs \#PCS-201-018, American Type Culture Collection, Manassas, VA) were cultured in DMEM (\#D6429, Sigma-Aldrich, St. Louis, MO) with $10 \% \mathrm{FBS}$ at $37^{\circ} \mathrm{C}$ in a humidified atmosphere of $5 \% \mathrm{CO} 2 .{ }^{11}$ The cells were cultured until $70 \%$ confluence on $100-\mathrm{mm}$ diameter culture dishes and then transferred to 6-well flat-bottom culture plates to perform the following experiments. Senescence of HGFs was induced by multiple passages or by stimulation with hydrogen peroxide $\left(\mathrm{H}_{2} \mathrm{O}_{2}\right) \cdot{ }^{12}$

\section{Senescence of HGFs induced by multiple passages}

HGFs at densities $2 \times 10^{5}$ cells per dish were seeded in 100-mm tissue culture dishes. The HGFs were passaged when the culture reached approximately $60-75 \%$ confluency. For SA- $\beta$-gal staining, 1,500 fibroblasts were placed in a $35 \times 10 \mathrm{~mm}$ dish for each passage. $10 \mu \mathrm{M}$ of the senolytic drug ABT263 (Navitoclax, Chemscine, CS-0013) was added to nine-generation passage-cultured HGFs and the cells were incubated for $24 \mathrm{~h}$. At the end of culture, total RNA was collected and the expression of p16, p21, IL$1 \beta$, and TNF-a was examined by real-time PCR.

\section{Senescence of HGFs induced by hydrogen peroxide}

At the 5 th passage, HGFs were placed in a $35 \times 10 \mathrm{~mm}$ dish. On day 0 , the cells were stimulated with $20 \mu \mathrm{M}$ hydrogen peroxide $\left(\mathrm{H}_{2} \mathrm{O}_{2}\right.$, Fujifilm Wako, Japan). ${ }^{12}$ At 5 days after stimulation with $20 \mu \mathrm{M} \mathrm{H}_{2} \mathrm{O}_{2}$, the cells were harvested for SA- $\beta$-gal staining, measurement of SA- $\beta$-gal activities, and real-time PCR.

\section{Animals}

Male $\mathrm{C} 57 / \mathrm{B} 6 \mathrm{~N}$ mice at the ages of 10 weeks and 20 months ( $\mathrm{n}=3$ per group) were used. The animals were bred in a strictly monitored air-conditioned clean room and were given standard laboratory pellets and water ad libitum at National Center of Geriatrics and Gerontology. The animals were sacrificed and gingival tissues were collected for real-time PCR and primary gingival fibroblast culture. All experimental procedures were reviewed and approved by National Center for Geriatrics and Gerontology. 
The protocol of this animal study was approved by the Research Facilities Committee for Laboratory Animal Science at National Center of Geriatrics and Gerontology (approval number 30-58) and performed in agreement with the ARRIVE guidelines.

\section{Immune staining of periodontal tissues from mice}

The oral mucosa around the upper maxillary molars was fixed and deparaffinized and then hydrated in xylene and ethanol. Before commencing with the IHC staining protocol, heat-mediated antigen retrieval was performed in the sections with citrate buffer $\mathrm{pH} 6$ for $5 \mathrm{~min}$. After blocking with normal rat serum for $30 \mathrm{~min}$ at room temperature, the sections were incubated with Anti-CDKN2A/p16INK4a antibody-Nterminal (1:500 diluent, ab189034, Abcam, Cambridge, UK), Anti-Iba1 antibody (1:400 diluent, ab178846, Abcam, Cambridge, UK), Anti-iNOS antibody (1:100 diluent, ab178945,Abcam, Cambridge, UK), and AntiMannose (CD206) Receptor antibody (1:500 diluent, ab64693,Abcam, Cambridge, UK) overnight at $4{ }^{\circ} \mathrm{C}$. $A B C$ biotin solution (Vectastain $A B C$ kit, Funakoshi Co., Ltd, Tokyo, Japan) and ABC reagent were subsequently used, and the sections were then developed using DAB substrate. ${ }^{13}$

\section{Primary mouse gingival fibroblast culture}

The oral mucosa around the upper maxillary molars was immediately washed in PBS and transferred to a culture dish. Mouse gingival fibroblasts (MGFs) were grown in DMEM supplemented with $10 \%$ FBS. The medium was changed every other day. The cells were grown to semi-confluence, harvested by trypsinization at $37^{\circ} \mathrm{C}$ for 3 min, and then subcultivated with DMEM supplemented with $10 \% \mathrm{FBS}$ in a new dish. In the experiment, cells that had been passaged twice were used as early-passage fibroblasts. ${ }^{14}$

\section{Measurements of SA- $\beta$-gal}

Cells in which senescence had been induced by multiple passages and stimulation with hydrogen peroxide were assessed by SA- $\beta$-gal staining using a senescence detection kit (Bio Vision, CA, USA) following the manufacturer's instructions. Cellular SA- $\beta$-gal activity was also evaluated using a 96-well cellular senescence assay kit (Cell Biolabs, San Diego, CA, USA) following the assay protocol. ${ }^{15}$

\section{Real-time PCR}

Total RNA was isolated from gingival tissues and HGFs with Nucleospin RNA (Takara Bio Inc., Shiga, Japan, cat no. U0955C) according to the manufacturer's instructions. The total RNA concentration was corrected to $100 \mathrm{ng} / \mu \mathrm{L}$ with a NanoDrop ${ }^{\text {rM }} 2000$ spectrophotometer (Thermo Fisher, Tokyo, Japan). Firststrand cDNA synthesis was carried out using a ReverTra Ace-a- kit (TOYOBO, Osaka, Japan). Real-time PCR was performed with FastStart Essential DNA Green Master (Roche, Mannheim, Germany) according to the manufacturer's protocol. One microgram of RNA was reverse-transcribed using the LightCycler96 System (Roche, Germany). The primer sequences used for each gene are shown in Table 1. 
Table 1

Sequences of primers used for real-time PCR

\begin{tabular}{|lll|}
\hline name & Forward & Reverse \\
\hline Human p16 & CTCGTGCTGATGCTACTGAGGA & GGTCGGCGCAGTTGGGCTCC \\
\hline Human p21 & CCGAAGTCAGTTCCTTGTGG & CATGGGTTCTGACGGACAT \\
\hline Human IL-1 $\beta$ & GCAGCCATGGCAGAAGTACCTGA & CCAGAGGGCAGAGGTCCAGGTC \\
\hline Human TNFa & AGG CGC TCC CCA AGA AGA CA & TCC TTG GCA AAA CTG CAC CT \\
\hline Mouse p16 & TGTCAGTGGTGGACCTGACCT & AGGGGAGATTCAGTGTGGTG \\
\hline Mouse p21 & GTGGGTCTGACTCCAGCCC & TTGAGCAGAAGAGCTGCTACGT \\
\hline Mouse iNOS & CGAAACGCTTCACTTCCAA & TGAGCCTATATTGCTGTGGGGCT \\
\hline Mouse CD206 & CTCTGTTCAGCTATTGGACGC & CGGAATTTCTGGGATTCAGCTTC \\
\hline Mouse MMP3 & CAAAACATATTTCTTTGTAGAGGACAA & TTCAGCTATTTGCTTGGGAA \\
\hline Mouse Col1a & CAATGGCACGGCTGTGTGCG & AGCACTCGCCCTCCCGTCTT \\
\hline Mouse Col4a & CAGATTCCGCAGTGCCCTA & GGAATAGCCGATCCACAGTGAG \\
\hline Mouse Col4a2 & GACCGAGTGCGGTTCAAAG & CGCAGGGCACATCCAACTT \\
\hline Mouse GAPDH & AACCTGCCAAGTATGATGA & GGAGTTGCTGTTGAAGTC \\
\hline
\end{tabular}

The samples were normalized to the housekeeping gene, GAPDH, and the results were reported for each sample relative to the control. The experiments were performed in triplicate for each condition. The value is presented as the fold change between the samples using the $\Delta \Delta \mathrm{Ct}$ method. ${ }^{13}$

\section{Statistical analysis}

Results of statistical analysis are expressed as means \pm SEM. The significance of differences between groups was examined using one-way analysis of variance (ANOVA) or two-tailed Student's t-test.

\section{Results}

\section{Senescent cells in gingival tissues of aged mice}

To investigate the state of cellular senescence in gingival tissues, we collected oral mucosa around the upper maxillary molars from aged mice (20-month-old mice) and we examined the expression levels of aging-related molecules (SA- $\beta$-gal, p16, and p21) in the tissues and compared them with those in tissues from young mice. First, we examined the mRNA expression levels of p16 and p21 by real-time PCR. The 
mRNA expression levels of p16 and p21 were increased in gingival tissues from aged mice compared to those in gingival tissues from young mice (Fig. 1A). Next, to investigate the localization of senescent cells in the tissues, we carried out immunohistochemical staining using an anti-p16 antibody. As shown in Fig. 1B, gingival tissues from aged mice were stained more strongly with the anti-p16 antibody than were gingival tissues from young mice. The staining was particularly strong in the granular layer and basement layer of the epithelium and in submucosal tissue of the aged tissues. On the other hand, in young mice, only the granular layer of gingival tissues was strongly stained. SA- $\beta$-gal expression was also increased in cells isolated from gingival tissues of aged mice compared to that in cells from gingival tissues of young mice (Fig. 1C). It is known that the expression and secretion of inflammatory cytokines are increased in senescent cells. ${ }^{16}$ Therefore, we compared the expression levels of IL-1 $\beta$ and TNF-a in gingival tissues from aged mice with those in gingival tissues from young mice. The mRNA expression levels of IL-1 $\beta$ and TNF- $\alpha$ were significantly increased in gingival tissues from aged mice compared to those in gingival tissues from young mice (Fig. 1D). It is known that the ratio of $M 1$ and $M 2$ macrophages (M1/M2 ratio) is altered in gingival tissue of chronic periodontitis. ${ }^{17}$ Therefore, we examined the dynamics of macrophages in the collected mouse gingival tissues. Staining of mouse gingival sections with anti-iNOS, anti-CD206, anti-lba-1 antibodies revealed that there was a large number of macrophages in gingival tissues from aged mice compared to that in gingival tissues from young mice (Fig. 1E). Next, we analyzed mRNA expression levels of iNOS (M1 marker) and CD206 (M2 marker) in gingival tissues by real-time PCR. The expression level of iNOS mRNA was higher in gingival tissues of aged mice than in gingival tissue of young mice. In contrast, the expression levels of CD206 mRNA were not different in gingival tissues of aged mice and young mice (Fig. 1E). To further investigate the relationship between aging and wound healing, we examined the expression of collagen in the same tissues. The mRNA expression levels of collagen $1 \mathrm{a} 1$, collagen $4 \mathrm{a} 1$, and collagen $4 \mathrm{a} 2$ were markedly reduced in gingival tissues from old mice compared to those in gingival tissue from young mice (Fig. 1F).

\section{Senescence of gingival fibroblasts in vitro}

Next,we investigated the properties of cellular senescence in gingival tissues in vitro. Cells are thought to undergo senescence by reaching the limit of the number of possible divisions. Cellular senescence is also induced by application of oxidative stress. Therefore, senescence properties of gingival tissue-derived cells were examined by long-term subculture of HGFs or by applying oxidative stress to the cells. First, senescence of cultured HGFs was assessed by SA- $\beta$-gal staining and measurement of SA- $\beta$-gal activity. A significant increase in the proportion of SA- $\beta$-gal cells was observed after 9 passages of gingival fibroblasts (Fig. 2A, B). The proportion of SA- $\beta$-gal cells continued to increase until passage 12, after which the cells stopped growing. An increase in SA- $\beta$-gal activity was also found in gingival fibroblasts after 10 passages (Fig. 2C).

Next, gingival fibroblasts were incubated for 5 days after addition of $20 \mu \mathrm{M}$ hydrogen peroxide, and the degree of senescence was evaluated by using SA- $\beta$-gal staining. A significant increase in the proportion of SA- $\beta$-gal positive cells was observed in the culture on day 5 after stimulation with $20 \mu \mathrm{M}$ hydrogen peroxide (Fig. 2D, E). We next examined the expression of p16 and p21, which are aging markers 
associated with the cell cycle, in long-term sub-cultured and hydrogen peroxide-stimulated gingival fibroblasts. Significant increases in the mRNA expression levels of p16 and p21 were observed in gingival fibroblasts after 10 and 11 passages (Fig. 2F, G). In addition, significant increases in p16 and p21 mRNA expression levels were confirmed in gingival fibroblasts stimulated with $20 \mu \mathrm{M}$ hydrogen peroxide (Fig. 2H, I).

Next, we investigated how the expression of inflammatory cytokines IL-1 $\beta$ and TNF- $\alpha$ was altered in gingival fibroblast cultures with increased senescent cells. The mRNA expression levels of IL-1 $\beta$ and TNFa were significantly increased in gingival fibroblasts passaged 9 times compared to those in cells passaged 3 times (Fig. 3A, B). IL-1 $\beta$ and TNF-a mRNA expression levels were also significantly increased in gingival fibroblasts stimulated with $20 \mu \mathrm{M}$ hydrogen peroxide for 5 days (Fig. 3C, D).

To elucidate the relationship between cellular senescence and expression of inflammatory cytokines, we analyzed changes in the expression of inflammatory cytokines when senescent cells were eliminated. Briefly, nine-generation passage-cultured HGFs were incubated with $10 \mu \mathrm{M}$ of ABT263 for $24 \mathrm{~h}$, and then the mRNA expression levels of $p 16, p 21, I L-1 \beta$, and TNF-a were examined. Treatment with ABT263 reduced the mRNA expression levels of p16 and p21 in nine-passaged HGF cultures to their expression levels in five-generation cultures (Fig. 4A, B). Furthermore, the mRNA expression levels of IL-1 $\beta$ and TNF-a, which was increased by nine-passaged cultures, were significantly decreased by the addition of ABT263 (Fig. 4C, D). These results suggest that the increased expression of proinflammatory cytokines by longterm passaged HGF culture is related to the increase in senescent cells in the culture.

\section{Discussion}

Periodontitis is a disorder for which the incidence increases with aging, and senescence is thought to be one of the risk factors of periodontitis. ${ }^{3}$ However, the relationship between senescence of periodontal tissues and pathogenesis of periodontitis has remained unclear. In this study, we analyzed gingival aging using a mouse model and cell culture models. We clarified that aging increases the number of senescent cells in the gingiva and enhances the expression of inflammatory cytokines, which may easily induce inflammation in periodontal tissue. Furthermore, the expression of those inflammatory cytokines was suppressed by removing senescent cells using a senolytic drug. The results suggested that periodontitis could be prevented and treated by regulating senescent cells in periodontal tissues.

Senescence is an important risk factor for lifestyle-related diseases such as arteriosclerotic diseases and diabetes and dementia. ${ }^{18}$ Periodontal disease is also a type of lifestyle-related disease ${ }^{19}$, and its relevance to systemic lifestyle-related diseases has been pointed out. Periodontal disease is also a disease for which incidence increases with aging and thus senescence is therefore to be a risk factor for its onset and progression. Periodontal tissue changes with aging. ${ }^{20}$ Aging of periodontal tissues results in significant anatomical and histological changes that cause decreased tissue homeostasis leading to faster disease progression and delayed tissue repair. ${ }^{21,22}$ It is also possible that resistance to infection decreases due to a decrease in immune function in elderly people, and this might be a reason why 
periodontal disease is more likely to develop. ${ }^{3}$ On the other hand, senescence is also observed in various cells constituting the organs of living organisms, and it has been reported that accumulation of senescent cells contributes to the decrease in function and to inflammation of each organ. ${ }^{23}$ Cellular senescence is a state in which somatic cells have stopped dividing and proliferating, and it has been shown that expression of cyclin-dependent kinase inhibitors (CDKIs) such as p16 and p21 is enhanced in cellular senescence. ${ }^{24,25}$ In this study, enhanced expression of p16 and p21 was confirmed in gingival tissues of aged mice and in gingival fibroblasts after multiple passages. These facts indicate that the cell cycle of fibroblasts constituting gingival tissues stops and the cells become senescent with aging. In addition, the increased expression of $\mathrm{p} 16$ and $\mathrm{p} 21$ is also caused by oxidative stress such as $\mathrm{H}_{2} \mathrm{O}_{2} \cdot{ }^{26-28}$ In this study, the mRNA expression levels of $\mathrm{p} 16$ and p21 in gingival fibroblasts were increased by stimulation with $\mathrm{H}_{2} \mathrm{O}_{2}$. Reactive oxygen species (ROS) are strongly induced by gram-negative bacteria such as periodontal pathogens. ${ }^{29}$ Therefore, sustained induction of ROS in periodontal tissue by periodontopathic plaque may also promote gingival aging. Increased secretion of various inflammatory cytokines, chemokines, and extracellular matrix-degrading enzymes from senescent cells has been observed, and it has been suggested that these factors cause chronic inflammation. ${ }^{30}$ In this study, we confirmed that aging-related markers were also expressed in oral mucosal tissues of aged mice and that the expression of inflammatory cytokines such as IL-1 $\beta$ and TNF- $\alpha$ was also increased in the tissues. Expression of inflammatory cytokines was also observed in gingival fibroblasts in which senescence was induced. Furthermore, their expression was suppressed by removing senescent cells. These results suggest that the expression of inflammatory cytokines is enhanced in relation to the increase in senescent cells. MMP-3 (stromelysin-1), which is one of Matrix metalloproteinases (MMPs), degrades collagen in the basement membrane as well as inducing the synthesis of other MMPs such as MMP-1 and MMP-9 and it potently destroys periodontal tissue. ${ }^{31}$ On the other hand, the association between aging of periodontal tissue-derived cells and expression of MMP-3 is unclear. In this study, the expression levels of MMP-3 mRNA was also enhanced in aged gingival fibroblast cultures (supplemental data \# 1). The enhanced expression of MMP-3 in senescent fibroblasts might be involved in the exacerbation of periodontitis.

The mRNA induction of inflammatory cytokines revealed in this study may cause SASP. It is also possible that the SASP factor acts on fibroblasts around senescent cells, leading to secondary induction of inflammatory cytokines and increasing their expression levels. These results suggest that the increased number of senescent cells in gingival tissue may form a condition that makes periodontal tissue more prone to inflammation by continuously producing inflammatory mediators. In this study, we also clarified that there was an increase in M1 macrophages in gingival tissues of aged mice along with an increase in total macrophages (Fig. 1E). Macrophages fall into the classical M1 and alternative M2 categories. M1 macrophages release various proinflammatory cytokines and are involved in enhancing the inflammatory response. On the other hand, M2 macrophages are thought to be involved in convergence of inflammation. In addition, changes in the balance of M1/M2 macrophages have been suggested to be involved in the development and progression of inflammation. ${ }^{32}$ Therefore, the increase in 
proinflammatory cytokines and M1 macrophages in the gingiva may be a mechanism for exacerbating inflammation and making it chronic. We speculate that this phenomenon may be the role of aging as a risk factor for periodontitis. In the future, we will confirm this phenomenon by conducting infection experiments with periodontopathic bacteria using aged mice.

The possibility of suppressing the onset of arteriosclerosis, dementia, and cancer by removing senescent cells has been revealed in a mouse model. ${ }^{33-36}$ Reagents that induce selective cell death of senescent cells (senolytic drugs) have been reported. ${ }^{37,38}$ ABT-263 is one of them and has been shown to induce cell death such as death of aging human fetal fibroblasts (MEFs). ${ }^{39}$ Therefore, we examined how the expression of inflammatory cytokines was changed by adding ABT-263 to gingival fibroblasts in which senescence was induced. ABT-263 attenuated the expression of hydrogen peroxide-induced cellular senescence markers and decreased the mRNA expression of IL-1 $\beta$ and TNF- $\alpha$ in the cultures. Therefore, it was thought that the increase in the expression of inflammatory cytokines by the addition of hydrogen peroxide to the cultures was due to an increase in senescent cells. In the future, it may be possible to prevent periodontal disease with senolytic drugs.

\section{Conclusion}

In this study, we confirmed that aging and oxidative stress increase senescent cells in mouse gingival tissues and gingival fibroblast cultures. The increase in senescent cells in the gingiva is caused sustained expression of inflammatory mediators and may contribute to the onset and chronicity of periodontitis.

\section{Declarations}

\section{Author Contributions}

All authors have made substantial contributions to conception and design of the study. MF, MK, JW and MW have been involved in data collection and data analysis. MF, Ka M, YS, YA and Ke M have been involved in data interpretation, drafting the manuscript and revising it critically and have given final approval of the version to be published.

\section{Acknowledgements}

This work was supported by National Geriatrics and Gerontology in Japan (Grant No.19-43).

The authors declare no potential conflicts of interest with respect to the authorship and/or publication of this article.

\section{References}

1 DALYs, G. B. D. \& Collaborators, H. Global, regional, and national disability-adjusted life-years (DALYs) for 315 diseases and injuries and healthy life expectancy (HALE), 1990-2015: a systematic 
analysis for the Global Burden of Disease Study 2015. Lancet 388, 1603-1658, doi:10.1016/S01406736(16)31460-X (2016).

2 Darveau, R. P. Periodontitis: a polymicrobial disruption of host homeostasis. Nat Rev Microbio/8, 481-490, doi:10.1038/nrmicro2337 (2010).

3 Ebersole, J. L. et al. Aging, inflammation, immunity and periodontal disease. Periodontol 200072 , 54-75, doi:10.1111/prd.12135 (2016).

4 Coppe, J. P., Desprez, P. Y., Krtolica, A. \& Campisi, J. The senescence-associated secretory phenotype: the dark side of tumor suppression. Annu Rev Pathol 5, 99-118, doi:10.1146/annurev-pathol121808-102144 (2010).

5 Hayflick, L. The Limited in Vitro Lifetime of Human Diploid Cell Strains. Exp Cell Res 37, 614-636, doi:10.1016/0014-4827(65)90211-9 (1965).

6 Kuilman, T., Michaloglou, C., Mooi, W. J. \& Peeper, D. S. The essence of senescence. Genes Dev 24, 2463-2479, doi:10.1101/gad.1971610 (2010).

7 Acosta, J. C. et al. Chemokine signaling via the CXCR2 receptor reinforces senescence. Cell 133, 1006-1018, doi:10.1016/j.cell.2008.03.038 (2008).

$8 \quad$ Kuilman, T. et al. Oncogene-induced senescence relayed by an interleukin-dependent inflammatory network. Cell 133, 1019-1031, doi:10.1016/j.cell.2008.03.039 (2008).

9 Wajapeyee, N., Serra, R. W., Zhu, X., Mahalingam, M. \& Green, M. R. Oncogenic BRAF induces senescence and apoptosis through pathways mediated by the secreted protein IGFBP7. Cell 132, 363-374, doi:10.1016/j.cell.2007.12.032 (2008).

10 Watanabe, S., Kawamoto, S., Ohtani, N. \& Hara, E. Impact of senescence-associated secretory phenotype and its potential as a therapeutic target for senescence-associated diseases. Cancer Sci 108, 563-569, doi:10.1111/cas.13184 (2017).

11 Hsu, T. N. et al. Targeting FAT1 Inhibits Carcinogenesis, Induces Oxidative Stress and Enhances Cisplatin Sensitivity through Deregulation of LRP5/WNT2/GSS Signaling Axis in Oral Squamous Cell Carcinoma. Cancers (Basel) 11, doi:10.3390/cancers11121883 (2019).

12 Kiyoshima, T. et al. Oxidative stress caused by a low concentration of hydrogen peroxide induces senescence-like changes in mouse gingival fibroblasts. Int J Mol Med 30, 1007-1012, doi:10.3892/ijmm.2012.1102 (2012).

13 Yamada, K., Matsushita, K., Wang, J. \& Kanekura, T. Topical Glucose Induces Claudin-1 and Filaggrin Expression in a Mouse Model of Atopic Dermatitis and in Keratinocyte Culture, Exerting Anti- 
inflammatory Effects by Repairing Skin Barrier Function. Acta Derm Venereo/ 98, 19-25, doi:10.2340/00015555-2807 (2018).

14 Iwayama, T. et al. Adiponectin regulates functions of gingival fibroblasts and periodontal ligament cells. J Periodontal Res 47, 563-571, doi:10.1111/j.1600-0765.2012.01467.x (2012).

$15 \mathrm{Yi}$, Z. et al. Effects of Nonsteroidal Anti-inflammatory Drugs on the Self-renewal Capacity of Blast Progenitors in Hematological Malignancies. Anticancer Res 37, 2315-2322, doi:10.21873/anticanres.11569 (2017).

16 Rodier, F. \& Campisi, J. Four faces of cellular senescence. J Cell Bio/ 192, 547-556, doi:10.1083/jcb.201009094 (2011).

17 Yang, J. et al. Enhanced activity of macrophage M1/M2 phenotypes in periodontitis. Arch Oral Biol 96, 234-242, doi:10.1016/j.archoralbio.2017.03.006 (2018).

18 Ferencz, B. \& Gerritsen, L. Genetics and underlying pathology of dementia. Neuropsychol Rev 25, 113-124, doi:10.1007/s11065-014-9276-3 (2015).

19 Genco, R. J. \& Borgnakke, W. S. Risk factors for periodontal disease. Periodontol 2000 62, 59-94, doi:10.1111/j.1600-0757.2012.00457.x (2013).

20 Van der Velden, U. Effect of age on the periodontium. J Clin Periodontol 11, 281-294, doi:10.1111/j.1600-051x.1984.tb01325.x (1984).

21 van der Velden, U. The onset age of periodontal destruction. J Clin Periodonto/ 18, 380-383, doi:10.1111/j.1600-051x.1991.tb02304.x (1991).

22 Tsalikis, L. The effect of age on the gingival crevicular fluid composition during experimental gingivitis. A pilot study. Open Dent J 4, 13-26, doi:10.2174/1874210601004010013 (2010).

23 Ovadya, Y. et al. Impaired immune surveillance accelerates accumulation of senescent cells and aging. Nat Commun 9, 5435, doi:10.1038/s41467-018-07825-3 (2018).

24 Baker, D. J. et al. Clearance of p16Ink4a-positive senescent cells delays ageing-associated disorders. Nature 479, 232-236, doi:10.1038/nature10600 (2011).

25 Baker, D. J. et al. Naturally occurring p16(Ink4a)-positive cells shorten healthy lifespan. Nature 530, 184-189, doi:10.1038/nature16932 (2016).

26 Chen, L. et al. 1,25-Dihydroxyvitamin D exerts an antiaging role by activation of Nrf2-antioxidant signaling and inactivation of p16/p53-senescence signaling. Aging Cel/ 18, e12951, doi:10.1111/acel.12951 (2019). 
27 Macip, S. et al. Inhibition of p21-mediated ROS accumulation can rescue p21-induced senescence. $E M B O \mathrm{~J}$ 21, 2180-2188, doi:10.1093/emboj/21.9.2180 (2002).

28 Tabasso, A. F. S., Jones, D. J. L., Jones, G. D. D. \& Macip, S. Radiotherapy-Induced Senescence and its Effects on Responses to Treatment. Clin Oncol (R Coll Radiol) 31, 283-289, doi:10.1016/j.clon.2019.02.003 (2019).

29 Damgaard, C. et al. Porphyromonas gingivalis-induced production of reactive oxygen species, tumor necrosis factor-alpha, interleukin-6, CXCL8 and CCL2 by neutrophils from localized aggressive periodontitis and healthy donors: modulating actions of red blood cells and resolvin E1. $J$ Periodontal Res 52, 246-254, doi:10.1111/jre.12388 (2017).

30 He, S. \& Sharpless, N. E. Senescence in Health and Disease. Cel/ 169, 1000-1011, doi:10.1016/j.cell.2017.05.015 (2017).

31 Sorsa, T., Tjaderhane, L. \& Salo, T. Matrix metalloproteinases (MMPs) in oral diseases. Oral Dis 10, 311-318, doi:10.1111/j.1601-0825.2004.01038.x (2004).

32 Sica, A. \& Mantovani, A. Macrophage plasticity and polarization: in vivo veritas. J Clin Invest 122, 787-795, doi:10.1172/JCl59643 (2012).

33 Matjusaitis, M., Chin, G., Sarnoski, E. A. \& Stolzing, A. Biomarkers to identify and isolate senescent cells. Ageing Res Rev 29, 1-12, doi:10.1016/j.arr.2016.05.003 (2016).

34 Bussian, T. J. et al. Clearance of senescent glial cells prevents tau-dependent pathology and cognitive decline. Nature 562, 578-582, doi:10.1038/s41586-018-0543-y (2018).

35 Kovacovicova, K. et al. Senolytic Cocktail Dasatinib+Quercetin (D+Q) Does Not Enhance the Efficacy of Senescence-Inducing Chemotherapy in Liver Cancer. Front Onco/ 8, 459, doi:10.3389/fonc.2018.00459 (2018).

36 Zhang, P. et al. Senolytic therapy alleviates Abeta-associated oligodendrocyte progenitor cell senescence and cognitive deficits in an Alzheimer's disease model. Nat Neurosci 22, 719-728, doi:10.1038/s41593-019-0372-9 (2019).

37 Zhu, Y. et al. The Achilles' heel of senescent cells: from transcriptome to senolytic drugs. Aging Cell 14, 644-658, doi:10.1111/acel.12344 (2015).

38 Kirkland, J. L., Tchkonia, T., Zhu, Y., Niedernhofer, L. J. \& Robbins, P. D. The Clinical Potential of Senolytic Drugs. J Am Geriatr Soc 65, 2297-2301, doi:10.1111/jgs.14969 (2017).

39 Chang, J. et al. Clearance of senescent cells by ABT263 rejuvenates aged hematopoietic stem cells in mice. Nat Med 22, 78-83, doi:10.1038/nm.4010 (2016). 

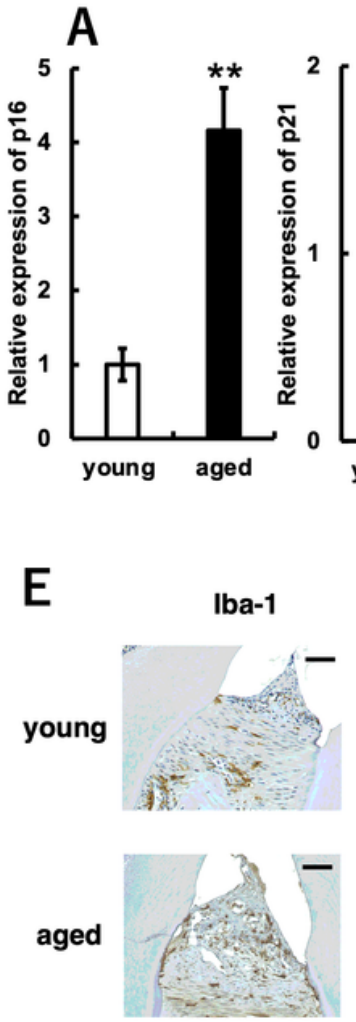

B

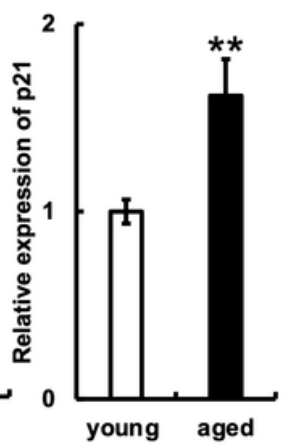

p16

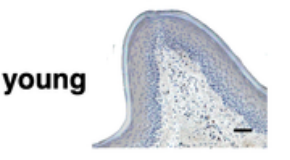

aged

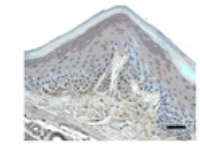

C

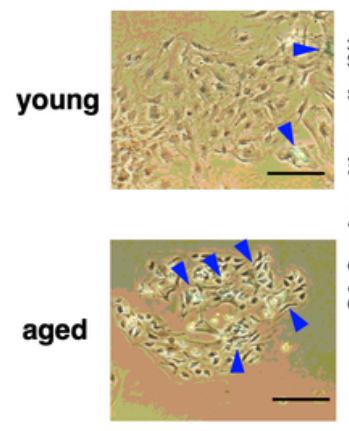

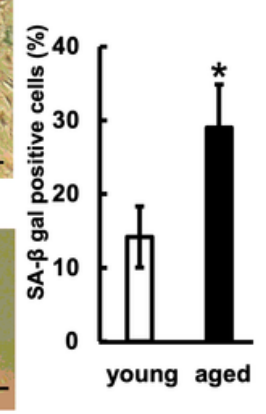
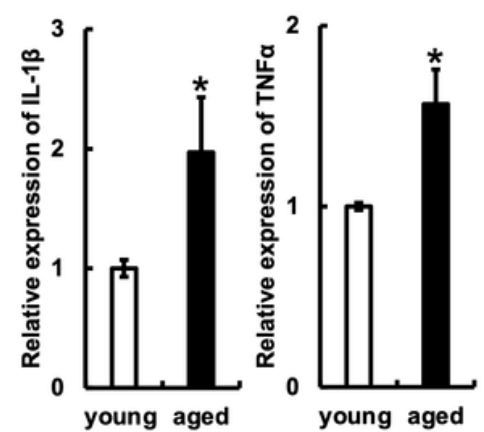

$\mathbf{F}$
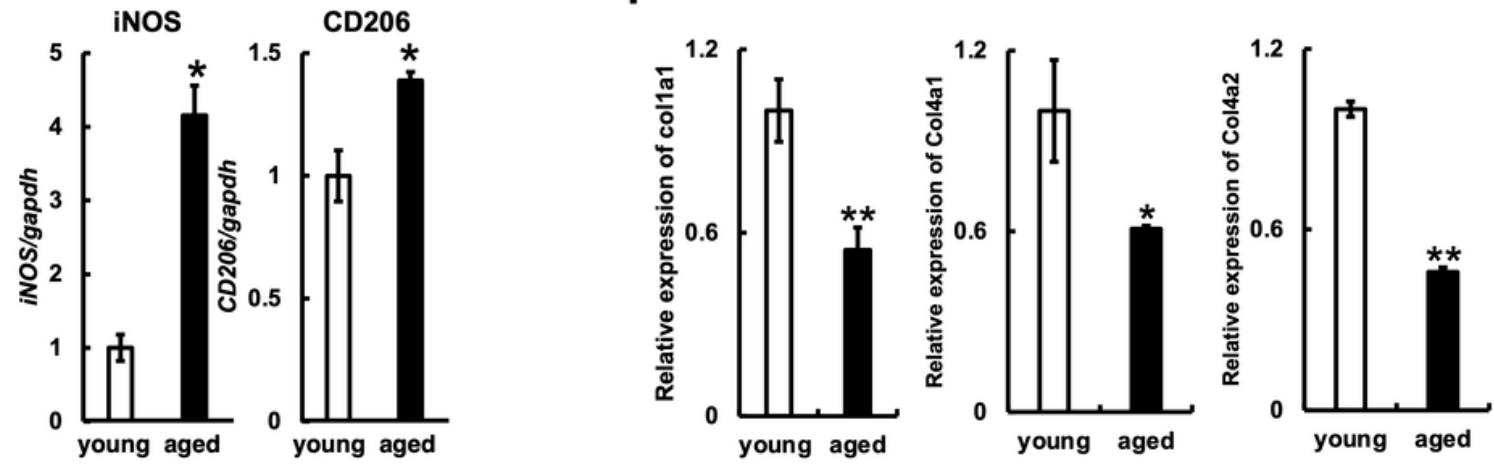

Figure 1

Senescent cells were increased in gingival tissues from aged mice. (A) Total RNA was isolated from gingival tissues of 6-week-old and 20-month-old male mice. The mRNA expression levels of p16 and p21 in the samples were measured by real-time PCR. (B) Gingival tissues were obtained from 6-week-old and 20-month-old male mice and paraffin sections were prepared. The production of p16 in the tissues was examined by immunostaining. Scare bars $=50 \mu \mathrm{m}$. (C) Fibroblasts isolated from gingival tissues of 6week-old and 20-month-old male mice were cultured in vitro. The degree of senescence of the cells passaged twice was evaluated by SA- $\beta$-gal staining. (D) Total RNA was isolated from gingival tissues of 6-week-old and 20-month-old male mice. The mRNA expression levels of IL-1 $\square$ and TNF- $\triangle$ in the samples were measured by real-time PCR. (E) Macrophages were increased in gingival tissues from aged mice. Areas of darkly stained epithelial tissue are shown. iNOS, CD206 and Iba1 staining x40. Scare bars $=50 \mu \mathrm{m}$. (F) Total RNA was isolated from gingival tissue of 6-week-old and 20-month-old male mice. The mRNA expression levels of collagen 1a1, 4a1, 4a2 in the samples were measured by real-time PCR. 
A
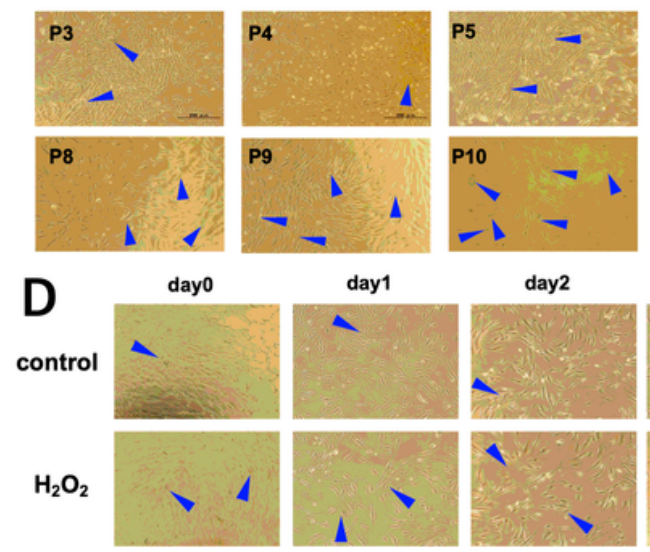

day2
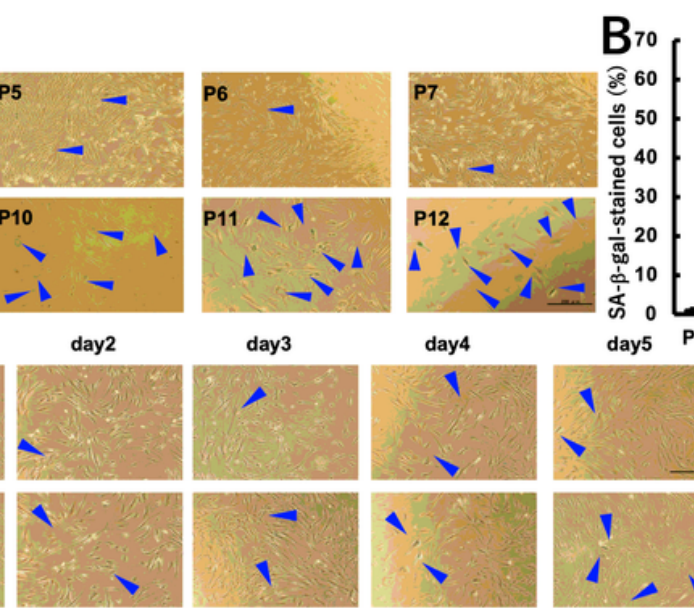

day3

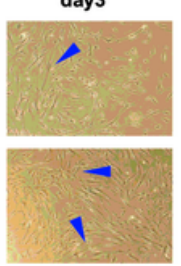

day 4

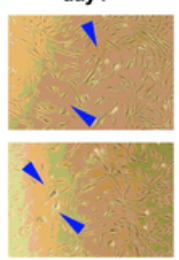

days

$\begin{array}{llllllllll}\text { P3 } & \text { P4 } & \text { P5 } & \text { P6 } & \text { P7 } & \text { P8 } & \text { P9 P10 P11 P12 }\end{array}$
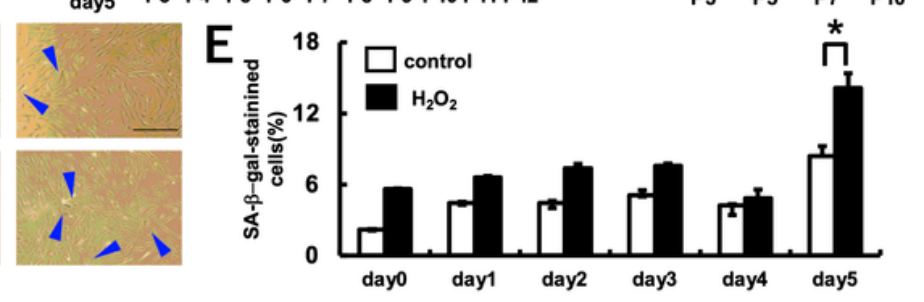
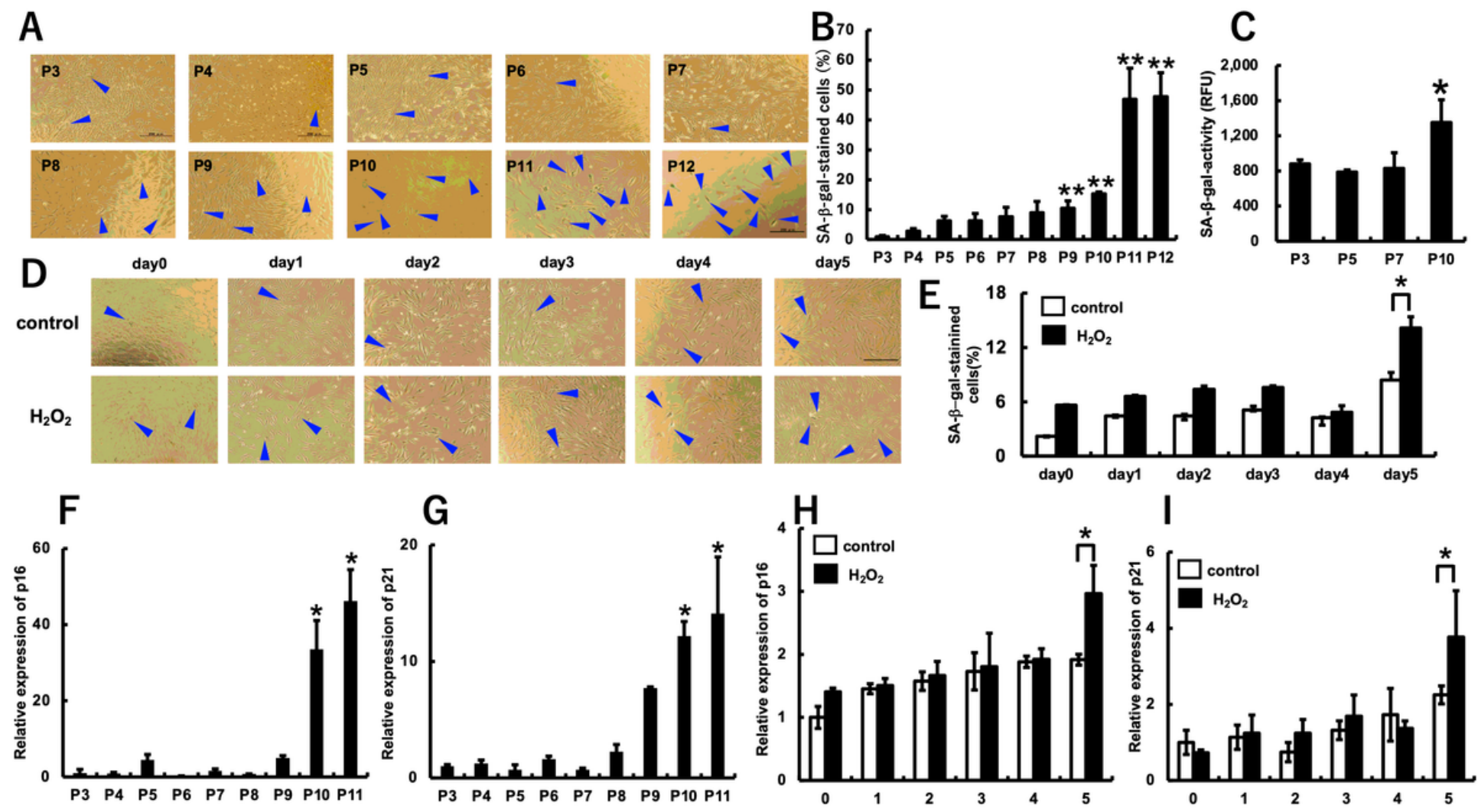

$\begin{array}{lllllllll}\text { P3 } & \text { P4 } & \text { P5 } & \text { P6 } & \text { P7 } & \text { P8 } & \text { P9 } & \text { P10 } & \text { P11 }\end{array}$

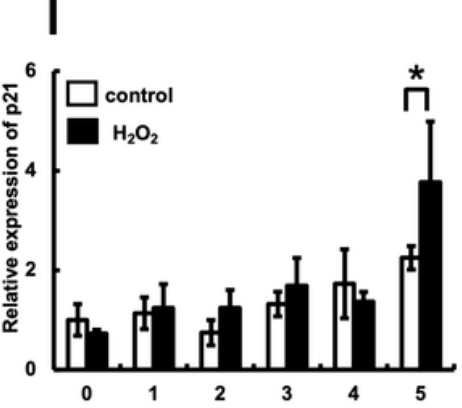

Figure 2

Analysis of cellular senescence of human gingival fibroblasts in culture. (A, B) Multiple passages of HGFs were executed in vitro. SA- $\beta$-gal staining was performed for HGFs of each passage to evaluate the degree of senescence. (C) SA- $\beta$-gal activities in HGFs of multiple passages were measured. (D, E) HGFs were treated with $20 \mu \mathrm{M} \mathrm{H} 2 \mathrm{O} 2$ for 5 days. SA- $\beta$-gal staining of HGFs was performed to evaluate the degree of senescence. The mRNA expression levels of p16 and p21 in multiple-passaged HGFs $(F, G)$ and $\mathrm{H} 2 \mathrm{O} 2-$ treated HGFs $(H, I)$ were measured by real-time PCR. 

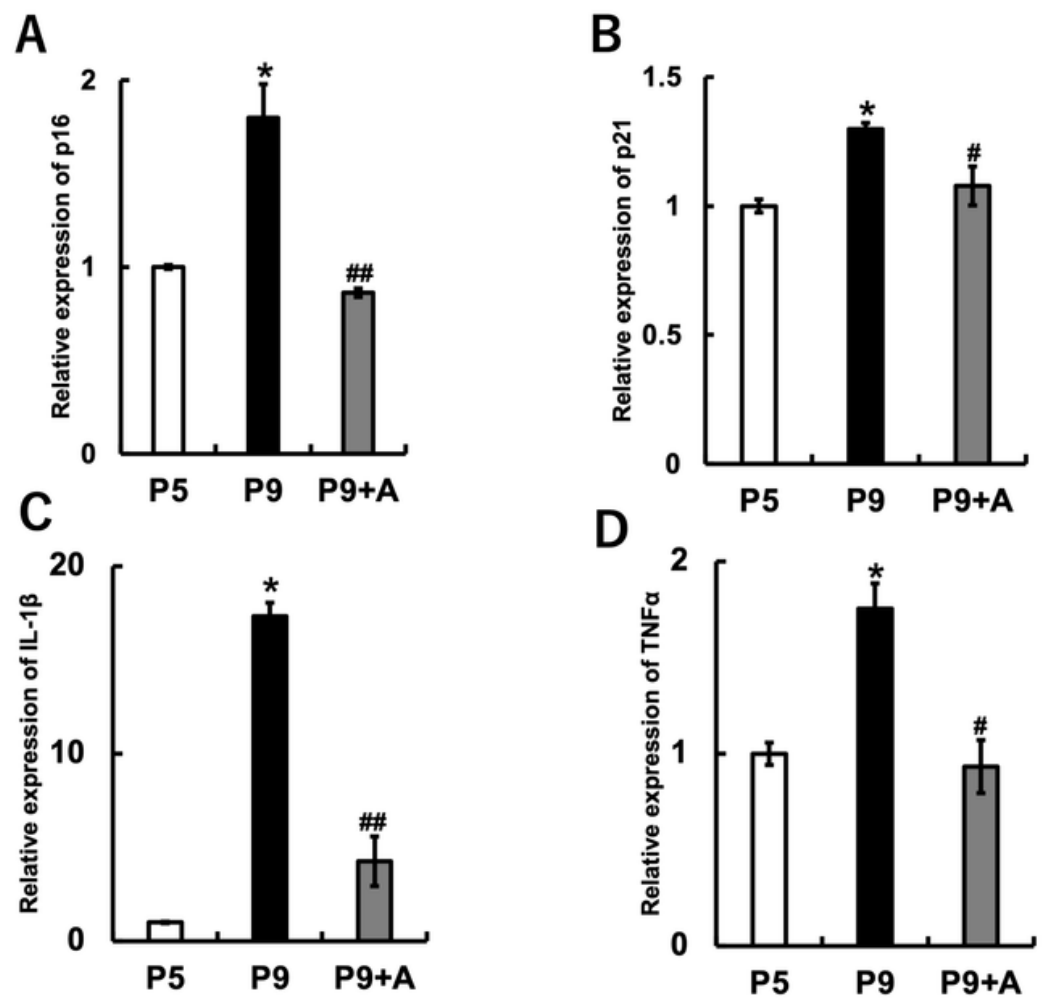

Figure 3

Inflammatory cytokines were increased in senescent gingival fibroblast cultures. The mRNA expression levels of IL-1 $\beta$ and TNF-aB $n$ 9th-passaged HGFs (A, B) and H2O2-treated HGFs (A, B) were measured by real-time PCR. 

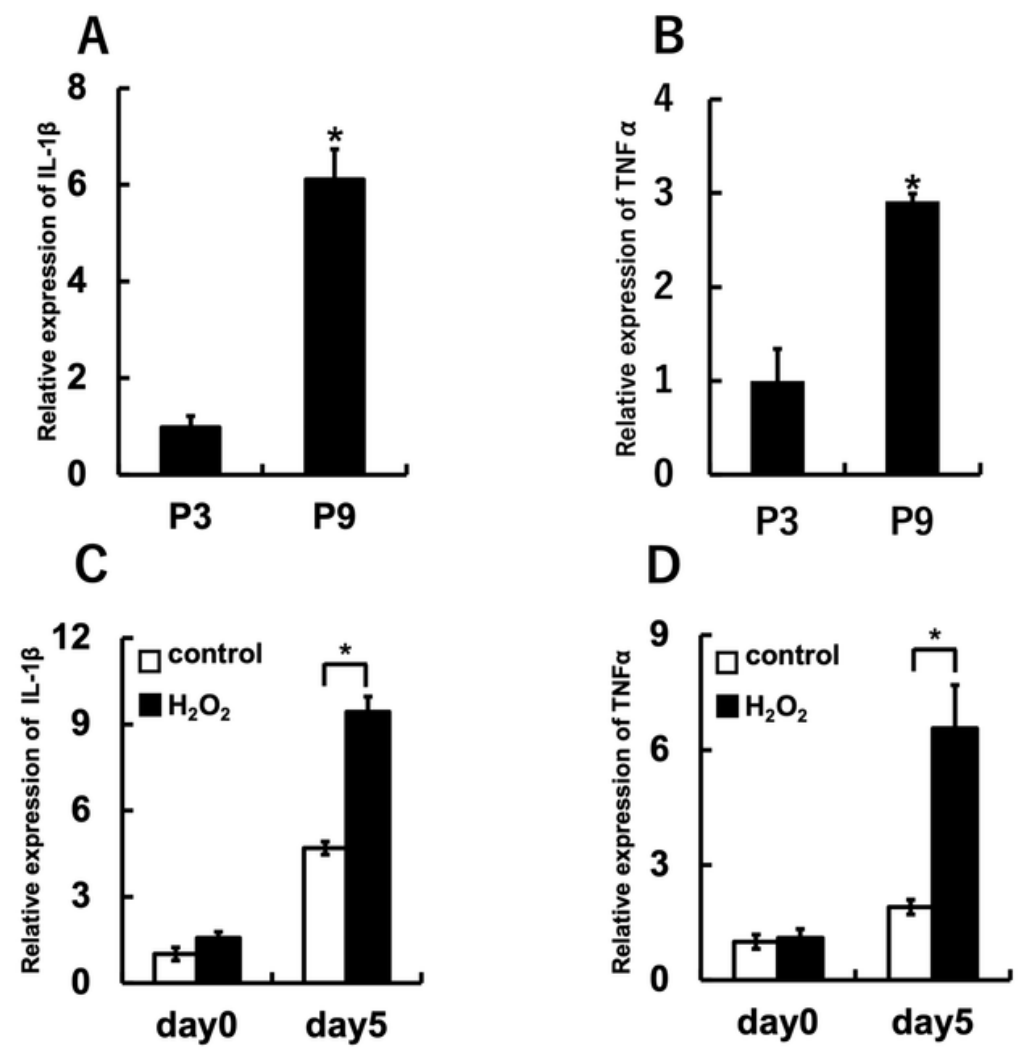

Fig. 3

\section{Figure 4}

Expression of inflammatory cytokines was suppressed by ABT263 in aged gingival fibroblast cultures. HGFs after 9 passages were incubated with $10 \mu \mathrm{M}$ of $A B T 263$ for $24 \mathrm{~h}$, and then the mRNA expression levels of p16, p21, IL-1 $\beta$, and TNF-a were examined by real-time PCR. A: ABT263.

\section{Supplementary Files}

This is a list of supplementary files associated with this preprint. Click to download.

- Supplementaldatainclud.authors.docx 